\title{
Surgery for Acute Stanford Type A Aortic Dissection in an Inner City Community Hospital: Single Surgeon's Experience
}

\author{
Jonathan Nwiloh \\ Section of Cardiothoracic Surgery, Atlanta Medical Center, Atlanta, GA, USA \\ Email: Jnwiloh@gmail.com
}

Received 21 January 2016; accepted 25 February 2016; published 29 February 2016

Copyright (C) 2016 by author and Scientific Research Publishing Inc. This work is licensed under the Creative Commons Attribution International License (CC BY). http://creativecommons.org/licenses/by/4.0/

(c) (i) Open Access

\section{Abstract}

Objective: An inverse relationship between volume and mortality in some cardiothoracic surgical procedures has been previously established, leading to suggestions that acute aortic dissection should not be operated in community or low volume heart centers. We therefore reviewed our experience to compare with published data. Methods: Retrospective review of 27 patients who underwent proximal aortic surgery by a single surgeon at an inner city community hospital between May 2004 and April 2015. 16 patients, mean age $51.7 \pm 13.6$ years old, $75.0 \%$ males underwent emergency surgery for acute Stanford type A aortic dissection, while 9 with root or ascending aortic aneurysm, mean age $50.3 \pm 15.0$ years old, $88.9 \%$ males had elective proximal aortic surgery. 2 patients with arch aneurysm were excluded. Results: Four $(25.0 \%)$ patients with acute dissection were in Penn class A, 3 (18.7\%) Penn B, 3 (18.7\%) Penn C and 6 (37.5\%) Penn B+C. 10 $(62.5 \%)$ patients underwent emergency root replacement with $60.0 \%(6 / 10)$ mortality all related to malperfusion including 2 patients with bloody stools, while $6(37.5 \%)$ underwent supracoronary graft replacement with $16.6 \%(1 / 6)$ mortality from cardiac tamponade. The 5-year survival was $89.0 \%$. In patients with aortic aneurysm, $8(88.9 \%)$ underwent elective root replacement and $1(11.1 \%)$ supracoronary graft replacement with zero mortality. Conclusion: Supracoronary graft replacement is performed for the majority of uncomplicated acute type A dissections and can be undertaken by the average general cardiac surgeon with acceptable results. Visceral malperfusion especially when associated with bloody stools portends a poor prognosis, and aortic dissection should be excluded in any Marfan patient presenting with acute abdomen. Delaying intervention in attempting transfer to a tertiary hospital can potentially increase preoperative mortality, known to rise with each passing hour from onset of acute dissection. Patients presenting therefore to community hospitals should probably undergo surgery there to avoid complications associated with delay. 
Keywords

\section{Acute Aortic Dissection, Surgical Outcome, Surgeon's Experience, Community Hospitals}

\section{Introduction}

Proximal acute aortic dissection remains one of the most dreaded emergencies in cardiothoracic surgery, often associated with comparatively higher mortality and morbidity than other commonly encountered pathologies in the specialty. These patients not infrequently present at night to community hospital based heart programs, some of whom may have limited experience with proximal aortic surgery. The estimated mortality for acute Stanford type A aortic dissection is high within the first 24 hours after onset. The surgeon at the community hospital may therefore be in a dilemma on whether to operate locally or attempt finding a specialized tertiary hospital that is willing to accept the patient. This can sometimes prove challenging especially with an uninsured patient in the US, with possible perception of dumping due to patient's inability to pay. The United States Congress in 1986 enacted a statute aimed at ensuring public access to emergency services regardless of ability to pay. This Emergency Medical Treatment and Labor Act (EMTALA) requires that a hospital treat and stabilize the emergency medical condition within its capability and capacity utilizing ancillary services including inpatient admission when necessary. Hospital and physician violators are subject to fines, exclusion from Government run Medicare and Medicaid programs, and potential lawsuits by patients. Recent advances in surgical techniques, cerebral protection strategies and the advent of hybrid endovascular aortic repairs for complicated dissections have all contributed to an overall improvement in survival over the last decade even in the hands of less experienced aortic surgeons.

\section{Materials and Methods}

Between May 2004 and April 2015, 27 patients operated by the author underwent proximal aortic surgery at an inner city community hospital. 16 patients presented with Acute Stanford Type A Aortic Dissection (AAAD) and 11 patients with root, ascending and arch aneurysm. The 2 patients with arch aneurysm were excluded from further analysis. Diagnosis of acute aortic dissection was usually made by computer tomography scanning (CTA) and confirmed by transesophageal echocardiogram (TEE) performed preoperatively or intraoperatively. Patients with aortic aneurysms also underwent elective cardiac catheterization. Patient characteristics and demographics are summarized in Table 1 \& Table 2. 10 (62.5\%) patients undergoing emergency surgery for AAAD were uninsured compared to none in the group undergoing elective aortic aneurysm surgery. Patients with AAAD were divided into two groups according to type of operation performed and for prognostication were also further categorized using the Penn classification based on clinical presentation (Table 3). All AAAD patients underwent emergency surgery with 10 (62.5\%) modified Bentall aortic root replacement with a mechanical valve conduit and $6(37.5 \%)$ supracoronary graft replacement with one concomitant coronary artery bypass surgery and one aortic valve repair. Patients with AAAD who underwent root replacement had either large root aneurysm, rupture or extension of dissection to the annulus with extensive destruction of the sinuses of valsalva. Patients with aortic aneurysms underwent elective repairs comprising of 8 (88.9\%) modified Bentall aortic root replacement with mechanical valve conduit and 1 (11.1\%) supracoronary graft replacement.

\section{Surgical Technique}

All surgeries were performed via a standard median sternotomy with arterial inflow through an $8 \mathrm{~mm}$ graft sewn end to side to the right axillary artery in acute aortic dissection patients, and either axillary, femoral or direct aortic cannulation in elective aortic aneurysm resection patients. Venous return was through a 2 stage cannula in the right atrium. The left ventricle was vented with a catheter placed through the right superior pulmonary vein after extracorporeal circulation was initiated. Once on cardiopulmonary bypass cooling was started while working on the proximal aorta after cardiac arrest with cold blood cardioplegia administered retrograde through the coronary sinus plus or minus antegrade. The diseased ascending aorta including the root when aneurysmal or destroyed was resected along with the intimal tear. Any prolapsed morphologically normal incompetent valve 
Table 1. Preoperative \& operative variables in acute aortic dissection group.

\begin{tabular}{|c|c|c|c|}
\hline Variable & Emerg. RR \#10 & Emerg. SCR \#6 & $\mathrm{p}$ value \\
\hline Mean age (years) & $46.4 \pm 9.5$ & $60.5 \pm 6.4$ & 0.04 \\
\hline Caucasian & $4(40.0 \%)$ & $1(16.6 \%)$ & 0.588 \\
\hline Male & $8(80.0 \%)$ & $4(66.7 \%)$ & 0.604 \\
\hline Cocaine use & $2(20.0 \%)$ & $1(16.7 \%)$ & 1 \\
\hline Hypertension & $6(60.0 \%)$ & $6(100.0 \%)$ & 0.234 \\
\hline Diabetes mellitus & 0 & $1(16.7 \%)$ & 0.375 \\
\hline COPD & $2(20.0 \%)$ & $1(16.7 \%)$ & 1 \\
\hline PVD & 0 & $1(16.7 \%)$ & 0.375 \\
\hline ESRD & $1(10.0 \%)$ & 0 & 1 \\
\hline Current smoker & $3(30.0 \%)$ & $1(16.7 \%)$ & 1 \\
\hline Marfan & $2(20.0 \%)$ & $1(16.7 \%)$ & 1 \\
\hline Root aneurysm & $7(70.0 \%)$ & $1(16.7 \%)$ & 0.119 \\
\hline Root size $(\mathrm{cm})$ & $5.9 \pm 1.3$ & $3.3 \pm 0.7$ & 0.006 \\
\hline Aortic rupture & $2(20.0 \%)$ & 2(33.3\%) & 0.604 \\
\hline Acute MI & $1(10.0 \%)$ & 0 & 1 \\
\hline AMS/CVA & $2(20.0 \%)$ & $1(16.7 \%)$ & 1 \\
\hline Limb paralysis & 0 & $2(33.3 \%)$ & 0.125 \\
\hline Bloody stool & $2(20.0 \%)$ & 0 & 0.5 \\
\hline Ejection fraction & $45.6 \pm 12.4$ & $47.5 \pm 2.7$ & 0.755 \\
\hline FHx. Root aneurysm & $2(20.0 \%)$ & 0 & 0.5 \\
\hline Pur. Pericarditis & $1(10.0 \%)$ & 0 & 1 \\
\hline СРB minutes & $352.6 \pm 45.2$ & $210.7 \pm 45.7$ & 0.001 \\
\hline ACP minutes & $231 \pm 40.8$ & $103.3 \pm 15.6$ & 0.001 \\
\hline Circ arrest minutes & $33.7 \pm 5.0$ & $31.3 \pm 9.0$ & 0.364 \\
\hline
\end{tabular}

RR = Root Replacement; SCR = Supracoronary Graft Replacement.

\section{Table 2. Preoperative \& operative variables in root replacement groups.}

\begin{tabular}{cccc}
\hline Variable & Gp. A Elect. RR \#8 & Gp. B Emerg. RR \#10 & p value \\
\hline MEAN AGE (YRS) & $49.6 \pm 15.9$ & $46.4 \pm 9.5$ & 0.6 \\
CAUCASIAN & $2(25.0 \%)$ & $4(40 \% 0$ & 0.638 \\
MALE GENDER & $8(100.0 \%)$ & $8(80.0 \%)$ & 0.477 \\
COCAINE USE & 0 & $2(20.0 \%)$ & 0.477 \\
HTN & $5(62.5 \%)$ & $6(60.0 \%)$ & 1 \\
DM & $2(25.0 \%)$ & 0 & 0.183 \\
COPD & $1(12.5 \%)$ & $2(20.0 \%)$ & 1 \\
CAD & $4(50.0 \%)$ & 0 & 0.023 \\
PVD & $2(25.0 \%)$ & 0 & 0.183 \\
ESRD & 0 & $1(10.0 \%)$ & 1 \\
CURRENT SMOKER & $1(12.5 \%)$ & $3(30.0 \%)$ & 0.588 \\
MARFAN & $1(12.5 \%)$ & $2(20.0 \%)$ & 1 \\
ROOT ANEURYSM & $8(100.0 \%)$ & $7(70.0 \%)$ & 0.216 \\
MEAN RA SIZE (CM) & 6.1 & 5.9 & 0.806 \\
AORTIC RUPTURE & 0 & $2(20.0 \%)$ & 0.477 \\
\hline
\end{tabular}




\begin{tabular}{cccc} 
Continued & & & \\
\hline ACUTE MI & 0 & $1(10.0 \%)$ & 1 \\
AMS/CVA & 0 & $2(20.0 \%)$ & 0.477 \\
FHX ROOT ANEURYSM & 0 & $2(20.0 \%)$ & 0.477 \\
BLOODY STOOL & 0 & $2(20.0 \%)$ & 0.477 \\
MEAN EF & $33.3 \pm 15.4$ & $45.6 \pm 12.4$ & 0.123 \\
PRIOR CARD. SURG. & $2(25.0)$ & 0 & 0.183 \\
PURU. PERICARDITIS & 0 & $1(10.0 \%)$ & 1 \\
CPB minutes & $292.8 \pm 31.6$ & $352.6 \pm 45.2$ & 0.013 \\
ACP minutes & $194.6 \pm 22.3$ & $231 \pm 40.8$ & 0.066 \\
CA minutes & $27.3 \pm 3.4$ & $33.7 \pm 5.0$ & 0.0381 \\
\hline
\end{tabular}

$\mathrm{RR}=$ Root Replacement.

Table 3. Penn classification for type a aortic dissection.

\begin{tabular}{cc}
\hline Penn Class & Clinical Presentation \\
\hline Class A & Absence of branch vessel malperfusion or circulatory collapse \\
Class B & Branch vessel malperfusion with ischemia \\
Class C & Circulatory collapse with or without cardiac involvement \\
Class B \& C & Branch vessel malperfusion and circulatory collapse \\
\hline
\end{tabular}

was resuspended to the commisures after the dissected aortic wall layers was reattached with a surgical adhesive BioGlue (CryoLife, Kennesaw, Georgia). The proximal graft anastomosis was then performed to the aorta reinforced with a strip of felt on the outside using 4 - 0 prolene (Ethicon Inc. Sommerville, New Jersey). In patients requiring root replacement, the valve conduit was attached to the aortic annulus with interrupted pledgeted 2 - 0 ethibond sutures (Ethicon Inc. Sommerville, New Jersey) and the coronary buttons reimplanted with a strip of felt to the graft using 5 - 0 prolene. The distal aortic anastomosis was performed during a period of circulatory arrest at nasopharyngeal temperature of 18 - 22 degrees Celsius, and the head packed with ice prior to arrest. Retrograde cerebral perfusion through a self inflating balloon catheter inserted in the superior vena cava at flows to maintain pressure of 15 - $20 \mathrm{mmHg}$ or unilateral antegrade cerebral perfusion through the right axillary artery graft at $6-10 \mathrm{ml} / \mathrm{kg} / \mathrm{min}$ to maintain perfusion pressure of $40-60 \mathrm{mmHg}$ with the innominate artery clamped were used as adjuncts in most patients during circulatory arrest. Intraoperative cerebral oximetry monitoring with Nonin SenSmart Model X-100 (Nonin Medical Inc. Plymouth, Minnesota) was used in some patients during the later years of the study. With patient in deep Trendelenburg the aortic cross clamp was released and the aorta trimmed appropriately before applying Bioglue to reattached the dissected layers. The open distal anastomosis between the glued aorta surrounded with a strip of felt on the outside and graft was performed using 4 - 0 prolene. BioGlue was also applied to each anastomosis with only small amounts around the proximal suture line in patients with a valve conduit. Mortality was regarded as death during the admission hospitalization or within 30 days of surgery. All data were entered into an excel spread sheet and imported into Sigma Plot (Systat Software, Inc. San Jose, CA) for statistical analysis. Categorical variables were reported as frequencies and percentages, while continuous variables were expressed as mean +/- standard deviations. The unpaired t- test and Fisher exact test were used for continuous and categorical variables respectively. $\mathrm{P}$ value $<0.05$ was considered statistically significant. Survival rates were estimated using the actuarial Kaplan Meier method.

\section{Results}

The 16 AAAD patients were aged 30 to 80 years, mean $51.7 \pm 13.6$ years with $75.0 \%$ (12) males, while the aortic aneurysm cohort comprised of 9 patients aged 23 to 71 years, mean $50.3 \pm 15.0$ years and $88.9 \%$ [8] males. In the AAAD cohort the mortality in patients undergoing emergency aortic root replacement was $60.0 \%(6 / 10)$ and in the supracoronary graft replacement patients $16.6 \%(1 / 6) . \mathrm{p}=0.145$. When the patients were further categorized by the Penn classification for risk stratification there was no mortality observed in class A patients. All 
the deaths occurred in patients with more complicated dissection according to the Penn classification. 2 patients presenting with signs of bowel ischemia and bloody stools died within 24 - 48 hours post operatively from refractory acidosis after aortic root replacement. Another patient presenting with acute severe aortic insufficiency, acute pulmonary edema, acute myocardial infarction and cardiogenic shock from involvement of both coronary arteries, and acute renal failure died 48 hours after aortic root replacement. The one death in the supracoronary graft replacement group occurred 2 weeks post operatively from cardiac tamponade enroute to the operating room for drainage. The causes of mortality are summarized in Table 4. One Marfan patient presenting with abdominal and back pain initially diagnosed as acute appendicitis (confirmed histologically with necrotic mucosa) underwent emergency laparoscopic appendectomy. Patient develop acute pulmonary edema within 24 hours post operatively and a stat transthoracic echocardiogram revealed acute type A aortic dissection confirmed on Chest CT scan which also showed a $7.5 \mathrm{~cm}$ root aneurysm with the dissection extending to the infra renal aorta. At emergency surgery for aortic root replacement he was found to have purulent pericarditis (Escherichia coli subsequently isolated), and patient did well post operatively except for prolonged ventilation. Two patients who presented with lower limbs paralysis underwent supracoronary graft replacement, with one remaining paraplegic and the second with only right lower limb paralysis improving postoperatively. The most frequently observed major morbidity was prolonged ventilation (Table 5). In the aneurysm cohort 8 underwent elective composite graft root replacement and 1 supracoronary graft replacement with $0.0 \%$ mortality compared to overall $43.8 \%$ (7/16) in the AAAD cohort. $\mathrm{P}=0.027$. The five year survival amongst the AAAD survivors was $89.0 \%$ (Figure 1).

\section{Discussion}

AAAD despite recent improvements in surgical techniques remains a formidable surgical challenge and it's presentation still causes a lot of anxiety amongst most cardiothoracic surgeons. With a low incidence of 2.9 in 100,000 per year [1], most surgeons will likely operate only on a handful of cases throughout their professional career except for perhaps those working in specialized aortic centers. Because of the limitations some have suggested referring these complex cases to tertiary hospitals with the surgical expertise, volume and experience in aortic surgery as a means of further improving surgical outcomes. This argument is supported by reviews such as that by Chikwe and colleagues [2]. In their recent US National outcome review of 5184 emergency repairs of acute aortic dissection between 2003 and 2008, they found that operative mortality was 27.5\% in lower

Table 4. Mortality based on Penn classification.
\begin{tabular}{ccccc}
\hline Cause & Class A \#4 & Class B \#4 & Class C \#3 & Class B+C \#6 \\
\hline Bleeding & 1 & & \\
Cardiac tamponade & 1 & & \\
Myocardial infarction & & 1 & 2 \\
Ischemic bowel & & & 2 \\
Multiorgan failure & & & \\
Mortality rate & 0 & $2(66.6 \%)$ & $1(33.3 \%)$ & $4(66.6 \%)$ \\
\hline
\end{tabular}

Table 5. Major morbidity for acute aortic dissection.

\begin{tabular}{ccccc}
\hline Major Complications & Penn A \#4 & Penn B \#3 & Penn C \#3 & Penn B+C \#6 \\
\hline Prolonged ventilation & 1 & 1 & 1 & 2 \\
Pneumonia & & 1 & 1 & 1 \\
ARF with dialysis & 1 & & \\
CVA & 1 & 1 & 4 \\
Reexploration for bleeding & 1 & & \\
Cardiac tamponade & & & 5 \\
Gangrene of toes & 1 & 4 & 5 \\
Total & & & \\
\hline
\end{tabular}




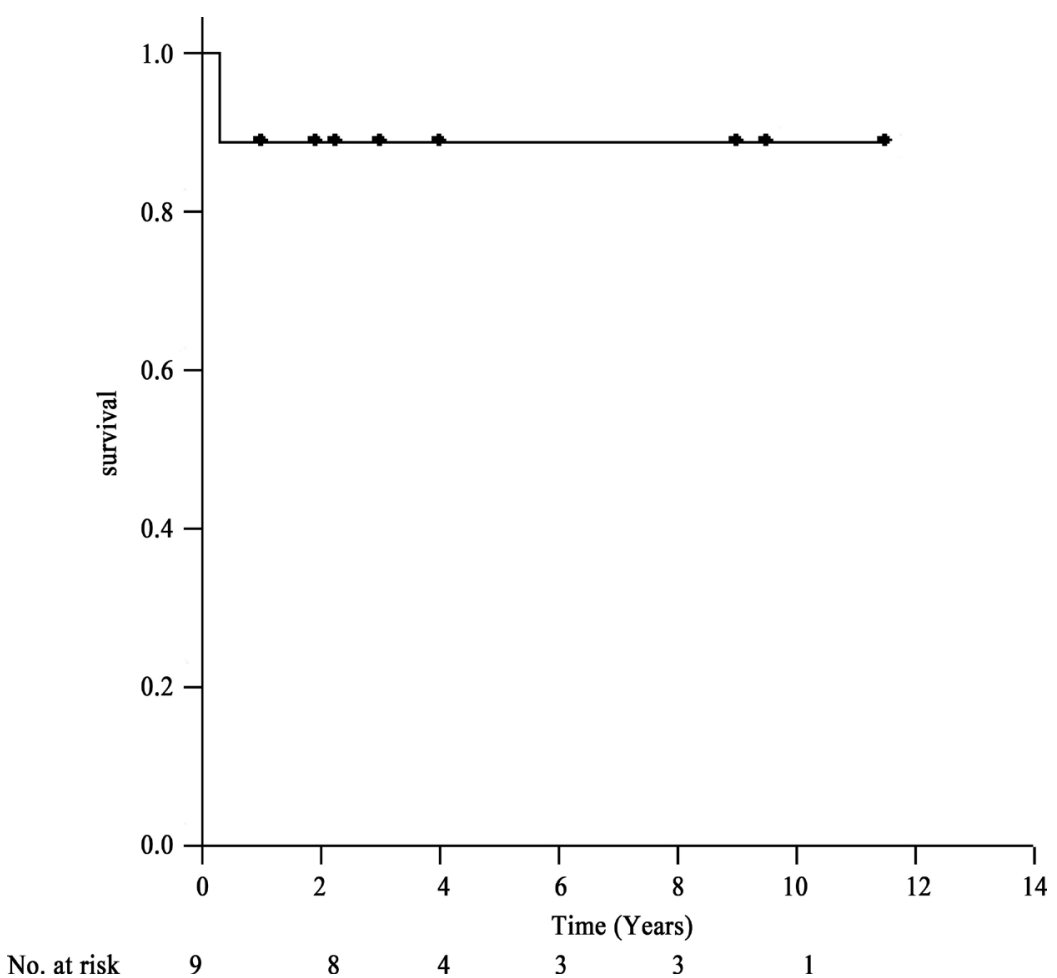

Figure 1. Kaplan Meier survival for aortic dissection patients.

volume surgeons (less than 1 case annually) compared to $17.0 \%$ in higher volume surgeons ( 5 or more annually). Similarly lower volume institutions ( 3 or fewer annually) had an operative mortality rate of $27.4 \%$ compared to $16.4 \%$ in high volume Institutions (more than 13 annually). Their study limitation includes been from an administrative database which did not include important variables such as type of surgical procedures performed, cardiopulmonary bypass, aortic cross clamp and circulatory arrest times. However a similar inverse relationship between increasing case volume and decreasing mortality pattern was observed by Hughes and colleagues [3] in their review of 13,358 patients in the Society of Thoracic Surgeons (STS) database undergoing elective aortic root replacement, aortic valve plus ascending aortic replacement and aortic valve sparing procedures from 2004 to 2007. They found an unadjusted operative mortality in the highest volume centers of $3.4 \%$ compared to $5.8 \%$ in the lowest volume centers. 534 (72\%) of the participating 741 centers were in the lowest volume group $(<6$ cases/year) and 22 (3\%) were in the highest volume group (30 - 100 case/year). Furthermore it's important to point out that the majority of single and multi-institutional reviews of emergency surgeries for AAAD in the literature are predominantly supracoronary graft replacement and the reported operative mortalities do not distinguish between aortic root replacement and supracoronary graft replacement groups. For instance the International Registry of Acute Aortic Dissection (IRAD) report by Trimarchi and colleagues [4] of surgical treatment for AAAD in 526 patients had an overall mortality of $25.1 \%$, with only $31.5 \%$ of the patients undergoing aortic root replacement. Similarly an Italian 7 tertiary multicenter study of 1148 AAAD patients from 1981 to 2013 reported an overall 30 day mortality of $25.7 \%$, with $30.0 \%$ of the patients undergoing Bentall or David root replacement [5]. Another US multi-institutional review of 251 patients from 2000 to 2010 undergoing emergency surgery for AAAD, reports only $23.0 \%$ having aortic root replacement [6]. Intuitively one would expect a somewhat higher operative mortality with aortic root replacement compared to supracoronary graft replacement especially in an emergency setting such as in AAAD. This has been demonstrated by the German Heart Center, Leipzig (A top European Aortic Surgery Center) series of 448 composite mechanical root replacement from 1998 to 2011. They reported an operative mortality of 3.9\% after elective/urgent surgery versus $20.0 \%$ after emergency surgery, about a 5 fold increase in mortality [7]. Also the sixth National United Kingdom Adult Cardiac Surgical database from 41 centers report from 2004 to 2008 identified 765 elective aortic root replacement surgeries with $8.0 \%$ mortality, versus $12.2 \%$ mortality in 213 urgent aortic root replacements [8].

From these two reports [7] [8] it is reasonable to assume therefore that the outcome of emergency aortic root 
replacement in the setting of AAAD is likely to be worse in the hands of the average general cardiac surgeon especially as most surgeons have limited experience with aortic root surgery. This is evident by the recent review of Stamou and colleagues [9] of the STS database of 13,746 patients who underwent aortic root surgery in the United States from 2004 to 2010. They found that most centers performed very small volumes, with a median of 2 annually per site and only 5\% of sites in the US performed more than 16 annually. This is probably why most advocate for the technically easier operation of supracoronary graft replacement in AAAD whenever possible especially in inexperienced hands. David [10] in a recent editorial on technical tips for the general cardiac surgeon confronted with operating emergently on AAAD, expressed the opinion that considering the increasing risk of death with each passing hour associated with this lethal disease, that it might be inappropriate to refer these cases to more experienced centers/surgeons because what might be gained in lower operative mortality could be offset by a higher preoperative mortality associated with delaying surgery. Moreover he states that even the most experienced surgeons can be humbled and do not always have the best outcome dealing with this disease. He further also advocates for supracoronary graft replacement of the dissected ascending aorta and only replacing the aortic root if the sinuses are aneurysmal or extensively dissected. One of the limitations of the STS database report by Hughes and colleagues [3] showing inverse relationship between surgical volume and operative mortality as pointed out by Soppa and colleagues [11] is the inability to adjust for individual surgeon volume, with results therefore possibly been due to either the high institutional volume or high surgeon volume.

Unlike the $20 \%$ - 30\% rate of aortic root replacement/remodeling for AAAD series in the literature, our small series, had a much higher rate, roughly $2 / 3$ (62.5\%) of patients requiring root replacement for the indications outlined above by Dr. Tirone David [10]. Our root replacement subset also had more complicated dissections, with 2 (20.0\%) in Penn class c and 5 (50.0\%) Penn class b +c. The University of Pennsylvania classification for AAAD based on clinical presentation has been shown to be useful in preoperative risk stratification with the worst surgical outcomes observed in patients with ischemic presentation [12]. This has been validated by others including Olsson and colleagues [13]. It is apparent that the most important determinant of mortality in addition to the complexity of the surgical procedure is the presence of a complicated aortic dissection at presentation. The poor outcome in the emergency root replacement subset in our series was therefore due to the ischemic presentation from malperfusion present in majority of these patients. With the emergence and now established benefit of thoracic endovascular aortic repair (TEVAR) as the procedure of choice for complicated Stanford Type B Aortic dissection [14] [15], and the more recent trend of hybrid or staged procedures with TEVAR of the descending thoracic aorta before or after ascending aortic replacement in patients with AAAD and malperfusion, the outlook for this group of patients has improved. From our experience bloody stools at presentation rarely reported in the literature appears to be a sign of severe mesenteric ischemia carrying an ominous prognosis with both patients having this finding dying postoperatively from severe refractory acidosis. Another observed unusual clinical ischemic presentation in our series was acute appendicitis in a Marfan patient presenting with abdominal and back pain, who underwent emergency laparoscopic appendectomy before decompensation from acute pulmonary edema leading to an echocardiogram showing severe aortic insufficiency from AAAD. The 43.8\% overall high mortality in our small AAAD series was a reflection of the high percentage of patients presenting with malperfusion and visceral ischemia. Girdauskas and colleagues [16] in their series of 276 patients with AAAD from Leipzig, observed a $29.0 \%$ mortality in 93 patients with malperfusion syndrome compared to $13.6 \%$ in 183 patients without malperfusion. However mortality was $75.0 \%$ (6/8) in the subgroup of patients (3.0\%) with mesenteric malperfusion. The 2014 European Society of Cardiology (ESC) guidelines also gives a Class IIA recommendation, Level of evidence B for TEVAR in AAAD with organ malperfusion to reduce mortality. It seems therefore at the present time that the mortality of patients with malperfusion syndrome especially mesenteric ischemia is high regardless of which Institution they are operated upon. The US review by Chikwe and colleagues [2] shows that $97.3 \%$ of the hospitals were urban, $78.2 \%$ were teaching hospitals, but only $13.9 \%$ were teaching with Thoracic surgical residency. So this means that about $1 / 5^{\text {th }}$ of cases approximately 1036 patients were treated at urban nonteaching hospitals during the 6 years review period. Given this finding, community hospitals will likely continue to provide emergency surgical treatment for AAAD in the US because of the established linear relationship of rising mortality with increasing time between onset and surgical intervention. This is further compounded by EMTALA act that penalizes inappropriate transfers when the expertise is available locally. There may also sometimes be reluctance by the other facility to accept the patient especially if uninsured when the hospital requesting transfer has a cardiac surgeon on their call roaster. Given that mortality in untreated patients with AAAD is reported to increase by $1 \%-3 \%$ per hour after presentation 
and up to $25 \%$ in 24 hours [17], unnecessary delays in operating may therefore expose the surgeon and hospital to punitive sanctions for EMTALA violation and possible law suit by the patients' families.

The study's limitations include the small number, retrospective nature and represent the experience of a single surgeon. The number however is reflective of what obtains in the vast majority of US hospitals with open heart surgery programs participating in the STS database, and is indicative of the real world experience of the average community based cardiac surgeon not infrequently faced with these challenging cases in the middle of the night.

\section{Conclusion}

In uncomplicated AAAD without malperfusion and ischemia, most community based general cardiac surgeons should be able to obtain good results with supracoronary graft replacement which is the operation of choice in the majority of patients presenting with this pathology. Emergency aortic root replacement in this setting is likely to carry a much higher mortality. In patients with evidence of visceral malperfusion especially with bloody stools, concomitant TEVAR at the time of ascending aortic replacement or staged shortly afterwards might be beneficial when feasible to help decrease mortality. Finally any Marfan patient presenting with acute abdomen must be ruled out for acute aortic dissection to allow early surgical intervention. Despite the small number of AAAD seen annually at most community hospitals, patients who first present at these institutions should probably undergo emergency surgery there as this may offer the best chance for patient survival.

\section{Conflict of Interest}

None.

\section{References}

[1] Meszaros, I., Morocz, J., Szlavi, J., Schmidt, J., Tornoci, L., Nagy, L., et al. (2000) Epidemiology and Clinicopathology of Aortic Dissection. Chest, 117, 1271-1278. http://dx.doi.org/10.1378/chest.117.5.1271

[2] Chikwe, J., Cavallaro, P., Itagaki, S., Seigerman, M., Diluozzo, G. and Adams, D. (2013) National Outcomes in Acute Aortic Dissection: Influence of Surgeon and Institutional Volume on Operative Mortality. The Annals of Thoracic Surgery, 95, 1563-1569. http://dx.doi.org/10.1016/j.athoracsur.2013.02.039

[3] Hughes, G., Zhao, Y., Rankin, J., Scarborough, J., O’Brien, S., Bavaria, J., et al. (2013) Effects of Institutional Volumes on Operative Outcomes for Aortic Root Replacement in North America. The Journal of Thoracic and Cardiovascular Surgery, 145, 166-170. http://dx.doi.org/10.1016/j.jtcvs.2011.10.094

[4] Trimarchi, S., Nienaber, C., Rampoldi, V., Myrmel, T., Suzuki, T., Mehta, R., et al. (2005) Contemporary results of surgery in acute type A aortic dissection: The International Registry of Acute Aortic Dissection experience. The Journal of Thoracic and Cardiovascular Surgery, 129, 112-122. http://dx.doi.org/10.1016/j.jtcvs.2004.09.005

[5] Russo, C., Mariscalco, G., Colli, A., Sante, P., Nicolini, F., Miceli, A., et al. (2015) Italian Multicenter Study on Type A Acute Aortic Dissection: A 33 Year Follow up. European Journal Cardio-Thoracic Surgery, 49, 125-131. http://dx.doi.org/10.1093/ejcts/ezv048

[6] Conway, B., Stamou, S., Kouchoukos, N., Lobdell, K., Khabbaz, K., Murphy, E., et al. (2014) Improved Clinical Outcomes and Survival Following Repair of Acute Type A Aortic Dissection in the Current Era. Interactive CardioVasc Thoracic Surgery, 19, 971-977. http://dx.doi.org/10.1093/icvts/ivu268

[7] Etz, C., Aspern, K., Girrbach, F., Battellini, R., Akhavuz, O., Leontyev, S., et al. (2013) Long Term Survival after Composite Mechanical Aortic Root Replacement: A Conservative Series of 448 Cases. The Journal of Thoracic and Cardiovascular Surgery, 145, S41-S47. http://dx.doi.org/10.1016/j.jtcvs.2012.11.045

[8] The Society for Cardiothoracic Surgery in Great Britain \& Ireland (2008) Sixth National Adult Cardiac Surgical Database Report 2008-Demonstrating Quality.

[9] Stamou, S., Williams, M., Gunn, T., Hagberg, R., Lobdell, K. and Kouchoukos, N. (2015) Aortic Root Surgery in the United States: A Report from the Society of Thoracic Surgeons Database. The Journal of Thoracic and Cardiovascular Surgery, 149, 116-122. http://dx.doi.org/10.1016/j.jtcvs.2014.05.042

[10] David, T. (2015) Surgery for Acute Type A Aortic Dissection. The Journal of Thoracic and Cardiovascular Surgery, 150, 279-283. http://dx.doi.org/10.1016/j.jtcvs.2015.06.009

[11] Soppa, G., Abdulkareem, N., Smelt, J., Van Besouw, J. and Jahangiri, M. (2013) High Volume Practice by a Single Specialized Team Reduces Mortality and Morbidity of Elective and Urgent Aortic Root Replacement. AORTA, 1, 4044. http://dx.doi.org/10.12945/j.aorta.2013.13.001

[12] Augoustides, J., Geirsson, A., Szeto, W., Walsh, E., Cornelius, B., Pochettino, A., et al. (2009) Observational Study of 
Mortality Risk Stratification by Ischemic Presentation in Patients with Acute Type A Aortic Dissection: The Penn Classification. Nature Reviews Cardiology, 6, 140-146. http://dx.doi.org/10.1038/ncpcardio1417

[13] Olsson, C., Hillebrant, C., Liska, J., Lockowandt, U., Ericksson, P. and Franco-Cereceda, A. (2011) Mortality in Acute Type A Aortic Dissection: Validation of the Penn Classification. The Annals of Thoracic Surgery, 92, 1376-1383. http://dx.doi.org/10.1016/j.athoracsur.2011.05.011

[14] Resch, T., Delle, M. and Falkenberg, M. (2006) Remodelling of the Thoracic Aorta after Stent Grafting of Type B Dissection: A Swedish Multicenter Study. The Journal of Cardiovascular Surgery, 47, 503-508.

[15] Sueyoshi, E., Sakamoto, I. and Hayashi, K. (2004) Growth Rate of Aortic Diameter in Patients with Type B Aortic Dissection during the Chronic Phase. Circulation, 110, 11256-11261. http://dx.doi.org/10.1161/01.CIR.0000138386.48852.b6

[16] Girdaukas, E., Kuntze, T., Borger, M., Falk, V. and Mohr, F. (2009) Surgical Risk of Preoperative Malperfusion in Acute Type A Aortic Dissection. The Journal of Thoracic and Cardiovascular Surgery, 138, 1363-1369. http://dx.doi.org/10.1016/j.jtcvs.2009.04.059

[17] Khan, I. and Nair, C. (2002) Clinical, Diagnosis and Management Perspectives of Aortic Dissection. Chest, 122, 311328. http://dx.doi.org/10.1378/chest.122.1.311 